\title{
Shaping liposomes by cell-free expressed bacterial microtubules
}

\author{
Johannes Kattan, Anne Doerr, Marileen Dogterom*, Christophe Danelon* \\ Department of Bionanoscience, Kavli Institute of Nanoscience, Delft University of Technology, van der
}

Maasweg 9, 2629 HZ, Delft, The Netherlands

*Correspondence: M.Dogterom@tudelft.nl; C.J.A.Danelon@tudelft.nl

\begin{abstract}
Genetic control over a cytoskeletal network inside lipid vesicles offers a potential route to controlled shape changes and DNA segregation in synthetic cell biology. Bacterial microtubules (bMTs) are protein filaments found in bacteria of the genus Prosthecobacter. They are formed by the tubulins BtubA and BtubB which polymerize in the presence of GTP. Here, we show that the tubulins BtubA/B can be functionally expressed from DNA templates in a reconstituted transcription-translation system, thus providing a cytosol-like environment to study their biochemical and biophysical properties. We found that bMTs spontaneously interact with lipid membranes and display treadmilling. When compartmentalized inside liposomes, de novo synthesized BtubA/B tubulins self-organize into cytoskeletal structures of different morphologies. Moreover, bMTs can exert a pushing force on the membrane and deform liposomes, a phenomenon that can be reversed by lightactivated disassembly of the filaments. Our work establishes bMTs as a new building block in synthetic biology. In the context of creating a synthetic cell, bMTs could help shape the lipid compartment, establish polarity or directional transport, and assist the division machinery.
\end{abstract}




\section{INTRODUCTION}

In eukaryotic cells, microtubules formed by the polymerization of $\alpha$ - and $\beta$-tubulin proteins are involved in essential functions, such as intracellular transport and chromosome segregation. Bacterial tubulin homologues have recently been discovered in Prosthecobacter species [1,2]. Called bacterial tubulin A and B (BtubA/B), these proteins interact to form microtubule-like structures in the presence of GTP [3]. Bacterial microtubules (bMTs) consist of five [4] or four protofilaments [5], as reported in in vivo and in vitro studies, respectively. Bacterial microtubules are thus noticeably thinner than eukaryotic microtubules which consist of 13 protofilaments. Recent in vitro studies have shown that bMTs exhibit dynamic instability (stochastic switching between growth and shrinkage) and treadmilling (apparent directional movement caused by the net addition of new subunits on one end and net removal on the other) $[5,6]$. These properties are also common to eukaryotic microtubules.

The function of bMTs remains unclear. Prosthecobacter itself belongs to the Phylum Verrucomicrobia and consists of Gram-negative bacteria which exhibit a high degree of compartmentalization. Prosthecobacter dejongeii, for example, possess a major membrane-bounded region, containing the fibrillar nucleoid and all the ribosome-like particles, as well as an intracytoplasmic membrane [7]. Another distinguishing feature of Prosthecobacter is the presence of narrowed extensions of the cell wall, called prosthecae [4]. Bacterial microtubules seem to be predominately located in these cell stalks, which suggests that they might be involved in their formation. It has also been proposed that BtubA/B filaments may contribute to intracellular organization [8]. The only currently known protein that interacts with bMTs is BtubC (also known as bacterial kinesin light chain, Bklc) which stabilizes bMTs [5] and links them to lipid membranes in vitro [8], suggesting it could play a role in anchoring BtubA/B filaments to membrane protrusions in vivo.

Unlike eukaryotic tubulin, BtubA/B is not dependent on protein chaperones and post-translational modifications, and can be functionally expressed in E. coli [4]. Because the cytoplasm of Prosthecobacter probably resembles that of E. coli [3], cell-free protein synthesis (CFPS) platforms derived from E. coli represent a physiologically relevant environment to investigate bMTs. Not only does CFPS allow to bypass protein purification, but it also enables the continuous interrogation of the protein dynamic behaviour in the course of its production [9].

To better apprehend the properties of bMTs in a cytosol-like environment, while taking advantage of the versatility of cell-free assays, we reconstituted in this study BtubA/B in a CFPS system. To further mimic the membrane-rich environment in Prosthecobacter, we characterized cell-free expressed BtubA/B on supported phospholipid bilayers and within vesicle compartments. Our results demonstrate that active BtubA/B proteins can be produced from their genes in vitro. Moreover, de novo synthesized bMTs can assemble on supported membranes and inside lipid vesicles (even without BtubC), where they form cytoskeletal structures that can deform the vesicle membrane. As CFPS inside liposomes has become an attractive platform to build a synthetic 
cell [10-17], we believe that bMTs could be exploited for spatial organization, polarization, and shape transformation of artificial cell models.

\section{RESULTS AND DISCUSSION}

We chose the PURE system [18], a reconstituted E. coli-based translation machinery (specifically the commercially available PUREfrex2.0), as our CFPS platform. This choice was motivated by the very low levels of protease and nuclease activity, and the wide range of (membrane) proteins synthesized in an active state with this system [12-20]. DNA templates for PUREfrex2.0 reactions consisted of the $b t u b A$ and $b t u b B$ genes from Prosthecobacter dejongeii. Both DNA constructs were sequence optimized for (i) expression in an E. coli host by matching codon occurrence with tRNA usage, (ii) low GC content within the first 30 base pairs (synonymous mutations were introduced to keep the amino acid sequence unaltered), and (iii) low propensity of intramolecular base-pairing of the messenger RNA around (in the vicinity or involving) the start codon (Supplementary Fig. 1). To satisfy the latter condition, the change in free Gibbs energy $(\Delta G)$ for a few RNA molecules was calculated. Lower $\Delta G$ values represent higher melting temperatures of the RNA molecule, which is known to be potentially inhibitory of translation in the PURE system [21]. Thus, we selected for each construct the DNA sequence whose corresponding mRNA has the predicted lowest melting temperature, assuming this would decrease the occurrence of inhibitory secondary structures involving the ribosome binding site and the start codon.

Proteins BtubA/B (Fig. 1A) were first separately expressed from linear DNA templates in PUREfrex2.0, supplemented with FluoroTect GreenLys reagent to fluorescently label gene products through co-translational incorporation of lysine residues conjugated to a fluorophore (Fig. 1B). Analysis of samples by denaturing polyacrylamide gel electrophoresis (PAGE) confirmed in vitro production of each Btub protein (Fig. 1C,

Supplementary Fig. 2). The concentration of expressed BtubA/B was estimated by using a standard curve with purified proteins where values of $\sim 13 \mu \mathrm{M}$ (BtubA) and $\sim 17 \mu \mathrm{M}$ (BtubB) were obtained (Supplementary Fig. 3). Subsequent co-expression from an equimolar amount of the $b t u b A$ and $b t u b B$ genes yielded $\sim 3 \mu \mathrm{M}$ of BtubA and $\sim 5 \mu \mathrm{M}$ of BtubB (Supplementary Fig. 3). It is unclear why the total concentration of synthesized proteins is lower in co-expression assays compared to separate expression of single genes.

Next, we examined the activity of purified and cell-free synthesized bMTs on supported lipid bilayers (SLBs). The membrane composition in SLB assays consisted of $\sim 50 \mathrm{~mol} \%$ of phosphatidylethanolamine (PE) and phosphatidylglycerol (PG) lipids, which are also found in the membrane of Prosthecobacter [22]. Purified BtubA/B proteins recombinantly expressed in E. coli cells were investigated in a PUREfrex2.0 background to closely emulate the molecular and ionic complexity of the bacterial cytosol. Bacterial microtubules were successfully assembled on top of an SLB from $2.5 \mu \mathrm{M}$ purified BtubA/B doped with Atto488-labelled subunits for visualization. The bMTs localized exclusively on the SLB and not on the bare glass areas (Fig. 2A). 
Filaments of BtubA/B appeared to stably interact with the membrane without the need for anchoring proteins such as BtubC. The protein BtubC synthesized in PUREfrex2.0 was able to bind to lipid membranes and to promote recruitment of BtubA/B (Supplementary Fig. 4 and Supplementary Note). Moreover, bMTs formed bundles of multiple protofilaments over time, suggesting lateral interactions. The critical BtubA/B concentration for the assembly of bMTs was reported to be $2.5-5 \mu \mathrm{M}$ at the potassium concentration present in the PURE system [6]. Here, bMTs were observed on SLBs already at concentrations of $\sim 1 \mu \mathrm{M}$ of purified tubulin in PUREfrex2.0 (Supplementary Fig. 5). This result suggests that, in the arguably more physiological conditions used here (in particular the higher molecular crowding), the critical concentration for BtubA/B polymerization is lower. Differences due to the types of activity assays (high-speed pelleting of BtubA/B and filaments grown from seeds in [6]) may also explain this change.

Single filaments appear to undergo directional movement along their longitudinal axis (Movie 1). This behaviour is presumably caused by simultaneous growth and shrinkage on the two opposite ends of the filament, a phenomenon known as treadmilling, as was previously also observed for purified BtubA/B filaments in simple buffers $[5,6]$. To validate this hypothesis, we used dual colour labelling of tubulin with the Atto488 or Atto565 dye, and bleached one of the fluorophores (Atto488) during imaging (Fig. 2B). Continuous illumination yielded filaments displaying extensions with fluorescent extremities originating from addition of tubulin subunits to the growing ends (Fig. 2C, D, Movie 2). Further, single fluorescent spots on the bleached filaments remained immobile. These observations demonstrate that bMTs undergo treadmilling behaviour on SLBs in a solution compatible with CFPS.

When cell-free expressed BtubA/B proteins, along with a trace amount of labelled purified bacterial tubulin (100 nM), were added to an SLB (Fig. 3A), assembly of bMTs and recruitment to the SLB were immediately observed (Fig. 3B). To rule out the possibility that filament assembly might be caused by the low amount of labelled purified tubulin, we also imaged the SLB with expressed BtubA and $100 \mathrm{nM}$ of labelled tubulin for $30 \mathrm{~min}$. No bMTs were observed in the absence of expressed BtubB, but readily appeared again upon addition of expressed BtubB (Fig. 3C, Movie 3). As with the purified proteins, dynamic instability, treadmilling on the membrane, as well as bundling of the microtubules were observed (Movie 3).

When the bMTs on the SLB were imaged at high illumination intensity, the filaments could break apart, either completely disassembling or with generated fragments detaching from the membrane (Fig. 2E). This effect was dependent on the presence of the fluorophore used for labelling (Atto488 or Atto565), suggesting a dye-specific photochemical reaction. If excitation was performed using a laser with a wavelength outside the excitation range of the fluorophore, prolonged illumination was not accompanied with filament breaking. Dual labelling experiments confirmed that the physical integrity of bMTs was altered, as opposed to photobleaching effects (Movie 4). 
Compartmentalization of bMTs in cell-sized lipid vesicles provides a unique platform to study their selforganization in a closed volume, as well as their ability to exert pushing forces and deform the membrane. Large and giant liposomes were produced by glass bead-assisted lipid film swelling [23,24]. PUREfrex 2.0 , the $b t u b A / B$ DNA constructs, a mix of DnaK chaperone to promote protein folding [9], and $100 \mathrm{nM}$ of Atto488-labelled purified BtubA/B were co-encapsulated (Fig. 4A). A slightly higher concentration of the $b t u b A$ gene compared to the $b t u b B$ gene ( $3.75 \mathrm{nM}$ vs $2.5 \mathrm{nM})$ was used to compensate for the lower amount of expressed BtubA vs BtubB when an equimolar concentration of the two templates is used (Fig. 1C). Liposomes that sedimented in a glass chamber were incubated at $37{ }^{\circ} \mathrm{C}$ to trigger gene expression and imaged at various time points by fluorescence confocal microscopy. Initially, liposomes that had encapsulated the labelled tubulin displayed an even fluorescence in their lumen (Supplementary Fig. 6). During the course of gene expression, filament structures appeared, with length and bundling propensity increasing over time (Fig. 4B, Supplementary Fig. 6). This internal cytoskeleton could clearly be attributed to in situ synthesis of BtubA/B as the small fraction of purified labelled tubulin did not yield filaments until accumulation of sufficient expression products (Supplementary Fig. 6) nor in control samples where the $b t u b A / B$ genes were omitted (Supplementary Fig. 7). The number of liposomes exhibiting cytoskeletal structures, the time of incubation until the first filaments appear (typically $1 \mathrm{~h}$ ), and the number of filaments per liposome, differed from one experiment to the other. Yet, the emergence of bMT networks was a robust observation. In the absence of DnaK mix, synthesized BtubA/B could not develop into cytoskeletal structures, suggesting that chaperones are needed to prevent synthesized proteins from aggregating or misfolding in liposomes [25]. A similar observation was made when expressing active Min proteins in PUREfrex2.0 [9]. A variety of BtubA/B filament and liposome morphologies was seen: straight or curved bundles or meshes, located across the lumen or near the membrane (Fig. 4C). Most liposomes containing bMTs exhibited morphological changes. Vesicles that were originally spherical showed local protrusions or global elongation (Fig. 4B, C). Such deformation events were likely the result of the pushing force exerted by growing bMTs on the liposome membrane, a phenomenon that is well documented for eukaryotic microtubules and actin filaments [26,27]. Similar results were obtained upon direct encapsulation of purified BtubA/B (6.6 $\mu \mathrm{M}$ final concentration in PUREfrex2.0), with the only noticeable difference that the average occurrence of filaments was higher with purified proteins, and a few cross-shaped liposomes were observed (Fig. 5). Liposome-to-liposome heterogeneity regarding the internal concentration of labelled tubulin, expression efficiency, and cytoskeleton features were likely the consequence of varying encapsulation efficiency and stochastic effects, as previously reported for other reconstituted biological systems $[9,12,16,17,23,24]$.

Finally, we sought to demonstrate the reversible nature of liposome deformations by the light-activated bMT disassembly mechanism that we observed on SLBs (Fig. 2E). Exposing filament-containing liposomes to intense illumination triggered bMT collapse and subsequent vesicle relaxation to a spherical shape (Fig. 4D). Similar observations have been reported with actin filaments [28]. In some cases, sphericality was not fully restored, which might be caused by high packing of liposomes or by surface tethering over an extended area, 
both effects altering the mechanical properties of the vesicles. Overall, these results demonstrate that liposomeconfined, de novo synthesized bMTs can self-organize into cytoskeletal structures that can deform the liposome through pushing forces on the membrane. Moreover, light-activated bMT disassembly can be exploited for reversible shape control.

To date, quantitative insights about the physical parameters describing bMT mechanical properties are missing. The bending rigidity of bMTs is probably lower than that of eukaryotic microtubules due to their smaller diameter $[4,5]$. The BtubA/B filament and bundle morphologies share similarities with those reported with encapsulated actin filaments bundled by linker proteins [29]. Therefore, we assume that the rigidity of bMTs lies in the range between eukaryotic microtubules and actin filaments. Other relevant parameters that remain to be determined include the critical force for buckling, the pushing force of a growing bMT, and the number of filaments per bundle. This information will help design liposome experiments, where membrane tension and BtubA/B expression levels may be adjusted to modulate the vesicle shape and organization of cytoskeletal structures.

This work expands the scope of bMT applications in synthetic biology. Bacterial microtubules have structural and biochemical properties that give them decisive advantages for bioengineering compared to eukaryotic microtubules. Their comparatively lower threshold of monomer concentration for polymerization, ability to form bundles without additional cofactors, and suitability for cell-free gene expression represent assets for implementation in artificial cells. Other prokaryotic protein filaments have already been reconstituted in liposomes. MreB expressed in liposomes assembles into cytoskeletal networks without inducing membrane deformation [30], unless molecular crowding at the vesicle membrane is applied [31]. Rings of FtsZ-FtsA can form budding necks and constrict liposomes [17]. Here, we show that bMTs expand the repertoire of protein filaments and range of associated functions. Endowing liposomes with a DNA-programmed mechanism for elongation and membrane remodelling, as realized here with bMTs, might be instrumental to support processes such as polarization and compartment division. For instance, bMT-mediated elongation of vesicles may promote pole-to-pole oscillations of the reconstituted Min system [9], or the positioning of the Z-ring [17]. The prospect for discovering new bMT-interacting partners (natural or engineered), especially end-tracking proteins, could further extend their role in intravesicular transport, segregation of synthetic chromosomes, or polarization. 


\section{MATERIALS AND METHODS}

\section{Preparation of DNA constructs}

To ensure a high expression level, we adjusted the DNA sequence of the three btub genes of Prosthecobacter dejongeii (btubA, btubB, btubC) with respect to their GC content and intramolecular base pairing for the first $30 \mathrm{bp}$ after the start codon. For each gene, we listed all possible DNA sequences of the first $30 \mathrm{bp}$ encoding the same amino acid sequence, selected the sequences with the lowest GC content and calculated the predicted melting temperature of their corresponding RNA sequence. We computed the conformations of the RNA sequences with the highest value for change in free Gibbs energy $(\Delta G)$ regarding the intramolecular base-pairing of the $30 \mathrm{bp}$ before and $30 \mathrm{bp}$ after the start codon (60 bp total) using mfold [32]. If sequences had similar $\Delta G$ values, the sequence with the least number of base-pairs at the ribosome binding site (RBS) and start codon was chosen. An example of the calculated RNA structures is shown in Supplementary Fig. 1. The sequenceoptimized DNA fragments, including a T7 promotor, RBS, and T7 terminator, were synthesized and cloned in the pUC57 plasmid (GenScript, United States). Linear templates were generated by PCR using forward and reverse primers 5'-CAGTCACGACGTTGTAAAACGAC-3' and 5'- CACACAGGAAACAGCTATGAC-3', respectively. The PCR products were purified using the Wizard SV Gel and PCR Clean-Up System (Promega), following the manufacturer's protocol. Concentration and purity of the DNA constructs were determined by spectrophotometry using the NanoDrop 2000 (Thermo Scientific). Samples were also analysed by electrophoresis on $1 \%$ agarose gels.

\section{Purification and labelling of BtubA/B}

BtubA/B proteins were purified and labelled as described in [6] except that C41(DE3) cells were used for expression instead of BL21(DE3). Concentration of purified bacterial tubulin was determined from absorbance at $280 \mathrm{~nm}$ (extinction coefficient $103754.2 \mathrm{M}^{-1} \mathrm{~cm}^{-1}$ ).

\section{Bulk CFPS}

Bulk CFPS reactions were performed with PUREfrex2.0 (GeneFrontier Corporation) and $5 \mathrm{nM}$ of linear DNA template according to the supplier's protocol. When $b t u b A$ and $b t u b B$ genes were co-expressed, $2.5 \mathrm{nM}$ of each DNA construct was used. For visualization of gene products by PAGE, $1 \mu \mathrm{L}$ of GreenLys solution (FluoroTect, Promega) was supplemented to a PUREfrex2.0 mix (total volume $10 \mu \mathrm{L}$ ) and the reactions were carried out in PCR tubes at $37{ }^{\circ} \mathrm{C}$ for $3 \mathrm{~h}$. Samples with added sodium dodecyl sulfate (SDS, 20\% w/v final) were incubated at $90{ }^{\circ} \mathrm{C}$ for $10 \mathrm{~min}$ and loaded on a $12 \%$ SDS-PAGE gel that was run first for $20 \mathrm{~min}$ at $100 \mathrm{~V}$ and then for 40 $\min$ at $160 \mathrm{~V}$. Fluorescently labelled proteins were visualized on a fluorescence gel imager (Typhoon, Amersham Biosciences) using a $488 \mathrm{~nm}$ laser and a 515-535-nm band-pass emission filter. Subsequently, the gel was stained with InstantBlue (expedeon) overnight and imaged with a ChemiDocTM imaging system (Bio$\mathrm{Rad})$. 


\section{Estimation of the concentration of synthesized BtubA/B}

Pre-ran PUREfrex 2.0 samples containing expressed bacterial tubulin were loaded onto a $10 \%$ stain-free gel, either undiluted or 5-fold diluted. Purified bacterial tubulin was loaded in concentrations ranging from 0.125 $\mu \mathrm{M}$ to $8 \mu \mathrm{M}$ and a calibration curve was generated from these samples using Fiji [33] to quantify band intensities.

\section{Imaging chambers}

Experiments involving SLB or liposome imaging were carried out in self-made glass chambers. Three glass slides of $1 \mathrm{~mm}$ thickness were glued together with NOA 61 UV-glue (Norland Products). Several holes of 3 $\mathrm{mm}$ diameter were created with a diamond drill and a 150- $\mu \mathrm{m}$-thick coverslip (Menzel-Gläser) glued to the bottom with NOA 61. Before use, the chambers were cleaned by sequential washing steps of 10 min sonication each in chloroform/methanol (1:1 volume), 2\% Hellmanex III (Hellma), $1 \mathrm{M} \mathrm{KOH}$, ethanol and MilliQ water. In case of SLB experiments, the glass chambers were additionally treated every second experiment with acid piranha solution. For some liposome experiments, aluminium chambers were used, which were fabricated in the same manner as described for the glass chambers and were cleaned following the same protocol, except that the $\mathrm{KOH}$ and piranha treatments were omitted.

\section{Preparation of lipid-coated beads}

All stock lipids in chloroform were supplied by Avanti Polar Lipids, except for Texas Red 1,2-dihexadecanoyl$s n$-glycero-3-phosphoethanolamine, triethylammonium salt (DHPE-TexasRed), which was from Invitrogen. A lipid mixture consisting of $50 \mathrm{~mol} \%$ 1,2-dioleoyl-sn-glycero-3-phosphocholine (DOPC), $36 \mathrm{~mol} \%$ 1,2dioleoyl-sn-glycero-3-phosphatidylethanolamine (DOPE), 12 mol\% 1,2-dioleoyl-sn-glycero-3-phospho-(1'rac-glycerol) (DOPG), 2 mol\% 1',3'-bis[1,2-dioleoyl-sn-glycero-3-phospho]-sn-glycerol (18:1 cardiolipin), 0.2 mol\% DHPE-TexasRed and 1 mass\% 1,2-distearoyl-sn-glycero-3-phosphoethanolamine-N[biotinyl(polyethylene glycol)-2000] (DSPE-PEG-biotin), was prepared in a 10-mL round-bottom glass flask. A solution of $100 \mathrm{mM}$ rhamnose in methanol was added (2.5:1 chloroform-to-methanol volume ratio), followed by $0.6 \mathrm{~g}$ of $212-300-\mu \mathrm{m}$ glass beads (acid washed, Sigma Aldrich). The solvent was removed by rotary evaporation at $200 \mathrm{mbar}$ for $2 \mathrm{~h}$ at room temperature. The lipid-coated beads were aliquoted, desiccated overnight and stored under argon at $-20^{\circ} \mathrm{C}$.

\section{Preparation of SUVs}

Small unilamellar vesicles (SUVs) had the same lipid composition as described for the preparation of lipidcoated beads, except that DSPE-PEG-biotin was omitted. A lipid film of $500 \mu \mathrm{g}$ was formed at the bottom of a glass vial by gentle evaporation of chloroform. Lipids were resuspended with $400 \mu \mathrm{L}$ MilliQ water (1.25 mg $\mathrm{mL}^{-1}$ final concentration) and the solution was vortexed for $2 \mathrm{~min}$. Sample was extruded using a mini extruder 
(Avanti Polar Lipids) equipped with $250 \mu \mathrm{L}$ Hamilton syringes, two filters (drain disc $10 \mathrm{~mm}$ diameter, Whatman) and a polycarbonate membrane with pore size of $0.2 \mu \mathrm{m}$ (first extrusion) and $0.03 \mu \mathrm{m}$ (second extrusion). The SUV stock solution was stored at $-20{ }^{\circ} \mathrm{C}$ until use.

\section{SLB experiments}

Imaging glass chambers were treated with oxygen plasma (Harrick Plasma) for 15 min to activate the surface. Six microliters of SUV solution were added to the chamber and supplemented with $12 \mu \mathrm{L}$ of $6 \mathrm{mM} \mathrm{CaCl}_{2}$. The chamber was covered with a coverslip, placed on a 0.5 -mm thick adhesive silicone sheet (Life Technologies), and incubated for $30 \mathrm{~min}$ at $37^{\circ} \mathrm{C}$. The formed SLB was washed four times with MRB80 buffer $(80 \mathrm{mM} \mathrm{K}-$ Pipes, $4 \mathrm{mM} \mathrm{MgCl}_{2}, 1 \mathrm{mM}$ EGTA, pH 6.8) and incubated $10 \mathrm{~min}$ with $0.5 \mathrm{mg} \mathrm{mL}^{-1} \mathrm{k}$-Casein in MRB80 buffer. For experiments with purified BtubA/B, $20 \mu \mathrm{L}$ of a solution containing PUREfrex $2.0,0.05 \%(\mathrm{w} / \mathrm{v}$ ) methylcellulose, $4 \mu \mathrm{L}$ MRB80, $3 \mu \mathrm{L}$ MilliQ water, purified unlabelled and Atto488/Atto561-labelled BtubA/B, were added to the glass chamber. Imaging was performed at either 25 or $30^{\circ} \mathrm{C}$. For experiments with cell-free synthesized BtubA/B, $8.5 \mu \mathrm{L}$ of a pre-ran PUREfrex2.0 sample containing expressed BtubA was mixed with $1.5 \mu \mathrm{L}$ of $1 \mu \mathrm{M}$ labelled BtubA/B-Atto488, the sample was added to the SLB and imaged for $30 \mathrm{~min}$ at $30{ }^{\circ} \mathrm{C}$. Then, $5 \mu \mathrm{L}$ of a pre-ran PUREfrex 2.0 sample containing expressed BtubB was added during total internal reflection fluorescence (TIRF) imaging. The setup consisted of an Ilas2 system (Roper Scientific) on a Nikon Ti-E inverted microscope with a Nikon CFI Plan Apochromat $100 \times$ NA1.45 TIRF oil objective and two Evolve 512 EMCCD camera's (Photometrics) for simultaneous dual-acquisition. The system was operated with MetaMorph 7.8.8.0 (Molecular Device).

\section{CFPS in liposomes}

Twenty micrograms of lipid-coated beads were added to $20 \mu \mathrm{L}$ of swelling solution consisting of PUREfrex2.0, $1 \mu \mathrm{L}$ DnaK mix (GeneFrontier Corporation), $100 \mathrm{nM}$ Atto488-BtubA/B, and $3.75 \mathrm{nM}$ of btubA and $2.5 \mathrm{nM}$ of $b t u b B$ DNA constructs. The sample was incubated on ice for $2 \mathrm{~h}$ during which the tube was gently manually rotated a few times. Four freeze-thaw cycles were applied by dipping the tube into liquid nitrogen, followed by thawing at room temperature. An imaging glass chamber was incubated for 5 min with a mix of bovine serum albumin (BSA) and BSA-biotin (1:1 molar ratio, $1 \mathrm{mg} \mathrm{mL}^{-1}$, Thermo Fisher Scientific), followed by incubation with Neutravidin ( $1 \mathrm{mg} \mathrm{mL}^{-1}$, Sigma Aldrich). Next, $4 \mu \mathrm{L}$ of liposome sample along with $12 \mu \mathrm{L}$ of dilution buffer (PUREfrex2.0 Solution I and MilliQ water (7:4 volume ratio) supplemented with $83 \mathrm{mg} \mathrm{L}^{-1}$ proteinase $\mathrm{K}$ ) were added to the imaging chamber. Fluorescence imaging was performed with a confocal microscope (A1+ from Nikon, 100× oil immersion objective) using the 488- and 561-nm laser lines for excitation of Atto488BtubA/B and DHPE-TexasRed, respectively. Samples were incubated at $37^{\circ} \mathrm{C}$ during image acquisition. 


\section{ACKNOWLEDGEMENTS}

The authors thank Vladimir Volkov for assistance with establishing bacterial microtubules in the lab and for useful discussions. Microscopy measurements were performed at the Kavli Nanolab Imaging Center Delft. This work was financially supported by the Netherlands Organization for Scientific Research (NWO/OCW) through the 'NanoFront - Frontiers of Nanoscience' Gravitation grant and the 'BaSyC - Building a Synthetic Cell' Gravitation grant (024.003.019).

\section{COMPETING INTERESTS}

The authors declare no competing interests.

\section{SUPPLEMENTARY INFORMATION}

Supplementary Information is available for this paper: gene sequences, supplemental methods, figures, movies and a note. 


\section{REFERENCES}

[1] Rosati, G., Lenzi, P, and Franco, V. (1993) 'Epixenosomes': Peculiar epibionts of the protozoon ciliate Euplotidium itoi: Do their cytoplasmic tubules consist of tubulin? Micron 24, 465-471.

[2] Petroni, G., Spring, S., Schleifer, K.-Z., Verni, F., and Rosati, G. (2000) Defensive extrusive ectosymbionts of Euplotidium (Ciliophora) that contain microtubule-like structures are bacteria related to Verrucomicrobia. Proc. Natl. Acad. Sci. U. S. A. 97, 1813-1817.

[3] Sontag, C. A., Staley, J. T., and Erickson, H. P. (2005) In vitro assembly and GTP hydrolysis by bacterial tubulins BtubA and BtubB. J. Cell Biol. 169, 233-238.

[4] Pilhofer, M., Ladinsky, M. S., McDowall, A. W., Petroni, G., and Jensen, G. J. (2011) Microtubules in bacteria: Ancient tubulins build a five-protofilament homolog of the eukaryotic cytoskeleton. PLoS Biol. 9 , e1001213.

[5] Deng, X., et al. (2017) Four-stranded mini microtubules formed by prosthecobacter BtubAB show dynamic instability. Proc. Natl. Acad. Sci. U. S. A. 114, E5950-58.

[6] Díaz-Celis, C., et al. (2017) Bacterial tubulins A and B exhibit polarized growth, mixed-polarity bundling, and destabilization by GTP hydrolysis. J. Bacteriol. 199, e00211-17.

[7] Lee, K.-C., Webb, R. I., Janssen, P. H., Sangwan, P., Romeo, T., Staley, J. T., and Fuerst, J. A. (2009) Phylum Verrucomicrobia representatives share a compartmentalized cell plan with members of bacterial phylum Planctomycetes. BMC Microbiol. 9, 5.

[8] Akendengue, L., Trepout, S., Grana, M., Voegele, A., Janke, C., Raynal, B., Chenal, A., Marco, S., and Wehenkel, A. M. (2017) Bacterial kinesin light chain (Bklc) links the Btub cytoskeleton to membranes. Sci. Rep. 7, 45668.

[9] Godino, E., Noguera López, J., Foschepoth, D., Cleij, C., Doerr, A., Ferrer Castellà, C., and Danelon, C. (2019) De novo synthesized Min proteins drive oscillatory liposome deformation and regulate FtsA-FtsZ cytoskeletal patterns. Nat. Commun. 10, 4969.

[10] Noireaux, V., and Libchaber, A. (2004) A vesicle bioreactor as a step towards an artificial cell assembly. Proc. Natl. Acad. Sci. U. S. A. 101, 17669-17674.

[11] Luisi, P. L., Ferri, F., Stano, P. (2006) Approaches to semi-synthetic minimal cells: a review. Naturwissenschaften 93, 1-13.

[12] van Nies, P., Westerlaken, I., Blanken, D., Salas, M., Mencía, M., and Danelon, C. (2018) Selfreplication of DNA by its encoded proteins in liposome-based synthetic cells. Nat. Commun. 9, 1583. 
bioRxiv preprint doi: https://doi.org/10.1101/2021.06.13.448053; this version posted June 13, 2021. The copyright holder for this preprint (which was not certified by peer review) is the author/funder, who has granted bioRxiv a license to display the preprint in perpetuity. It is made available under aCC-BY-NC-ND 4.0 International license.

[13] Berhanu, S., Ueda, T., and Kuruma, Y. (2019) Artificial photosynthetic cell producing energy for protein synthesis. Nat. Commun. 10, 1325.

[14] Fujii, S., Matsuura, T., Sunami, T., Kazuta, Y., and Yomo, T. (2013) In vitro evolution of $\alpha$-hemolysin using a liposome display. Proc. Natl. Acad. Sci. U. S. A. 110, 16796-1680.

[15] Scott, A., Noga, M. J., de Graaf, P., Westerlaken, I., Yildirim, E., and Danelon, C. (2016) Cell-free phospholipid biosynthesis by gene-encoded enzymes reconstituted in liposomes. PLoS One 11, e0163058.

[16] Blanken, D., Foschepoth, D., Calaça Serrão, A., and Danelon, C. (2020) Genetically controlled membrane synthesis in liposomes. Nat. Commun. 11, 4317.

[17] Godino, E., Noguera López, J., Zarguit, I., Doerr, A., Jimenez, M., Rivas, G., and Danelon, C. (2020) Cell-free biogenesis of bacterial division proto-rings that can constrict liposomes. Commun. Biol. 3, 539.

[18] Shimizu, Y., Inoue, A., Tomari, Y., Suzuki, T., Yokogawa, T., Nishikawa, K., and Ueda, T. (2001) Cellfree translation reconstituted with purified components. Nat. Biotechnol. 19, 751-755.

[19] Doerr, A., Foschepoth, D., Forster, A. C., and Danelon, C. (2021) In vitro synthesis of 32 translation-factor proteins from a single template reveals impaired ribosomal processivity. Sci. Rep. 11:1898.

[20] Liu, Y. J., Hansen, G. P., Venancio-Marques, A., Baigl, D. (2013) Cell-free preparation of functional and triggerable giant proteoliposomes. Chembiochem 14, 2243-2247.

[21] Sadler, F. W., Dodevski, I., and Sarkar, C. A. (2018) RNA thermometers for the PURExpress system. ACS Synth. Biol. 7, 292-296.

[22] Lee, J., Park, B., Woo, S.-G., Lee, J., and Park, J. (2014) Prosthecobacter algae sp. nov., isolated from activated sludge using algal metabolites. Int. J. Syst. Evol. Microbiol. 64, 663-667.

[23] Nourian, Z., Roelofsen, W., and Danelon, C. (2012) Triggered gene expression in fed-vesicle microreactors with a multifunctional membrane. Angew. Chem. Int. Ed. Engl. 51, 3114-3118.

[24] Blanken, D., van Nies, P., and Danelon, C. (2019) Quantitative imaging of gene-expressing liposomes reveals rare favorable phenotypes. Phys. Biol. 16, 45002.

[25] Fernandez, C., and Giraldo, R. (2018) Modulation of the aggregation of the prion-like protein RepAWH1 by chaperones in a cell-free expression system and in cytomimetic lipid vesicles. ACS Synth. Biol. 7, 2087-2093.

[26] Hotani, H., and Miyamoto, H. (1990) Dynamic features of microtubules as visualized by dark-field microscopy. Adv. Biophys. 26, 135-156. 
bioRxiv preprint doi: https://doi.org/10.1101/2021.06.13.448053; this version posted June 13,2021 . The copyright holder for this preprint (which was not certified by peer review) is the author/funder, who has granted bioRxiv a license to display the preprint in perpetuity. It is made available under aCC-BY-NC-ND 4.0 International license.

[27] Cortese, J. D., Schwab, B. 3rd, Frieden, C., and Elson, E. L. (1989) Actin polymerization induces a shape change in actin-containing vesicles. Proc. Natl. Acad. Sci. U. S. A. 86, 5773-5777.

[28] Tanaka, S., Takiguchi, K., and Hayashi, M. (2018) Repetitive stretching of giant liposomes utilizing the nematic alignment of confined actin. Commun. Phys. 1, 18.

[29] Tsai, F.-C., and Koenderink, G. H. (2015) Shape control of lipid bilayer membranes by confined actin bundles. Soft Matter 11, 8834-8847.

[30] Maeda, Y. T., Nakadai, T., Shin, J., Uryu, K., Noireaux, V., and Libchaber, A. (2012) Assembly of MreB filaments on liposome membranes: a synthetic biology approach. ACS Synth. Biol. 1, 53-59.

[31] Garenne, D., Libchaber, A., and Noireaux, V. (2020) Membrane molecular crowding enhances MreB polymerization to shape synthetic cells from spheres to rods. Proc. Natl. Acad. Sci. U. S. A. 117, 1902-1909.

[32] Zuker, M. (2003) Mfold web server for nucleic acid folding and hybridization prediction. Nucleic Acids Res. 31, 3406-3415.

[33] Schindelin, J., Arganda-Carreras, I., Frise, E., Kaynig, V., Longair, M., Pietzsch, T., et al. (2012) Fiji: an open-source platform for biological-image analysis. Nat. Methods 9, 676-682. 


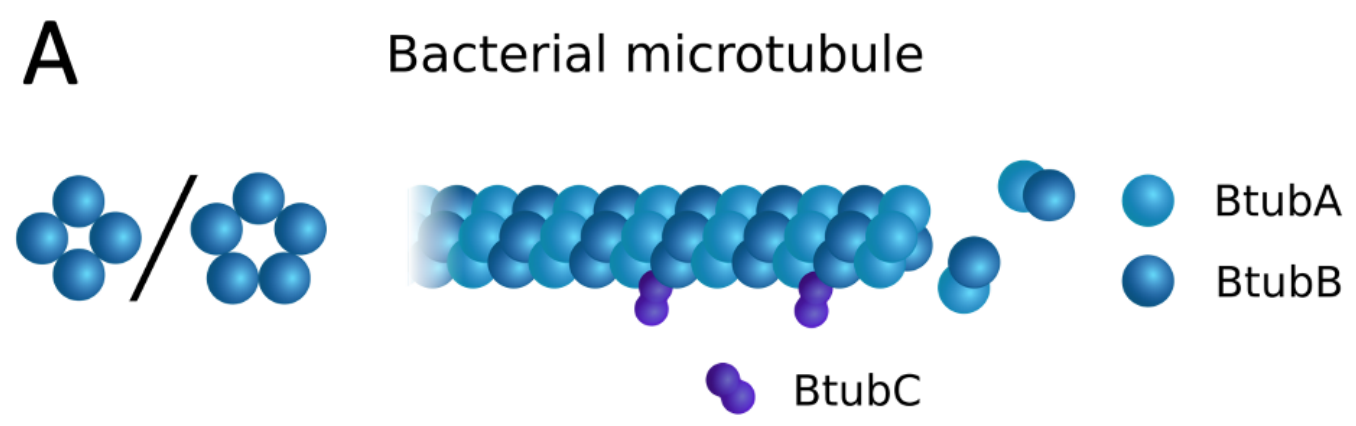

B
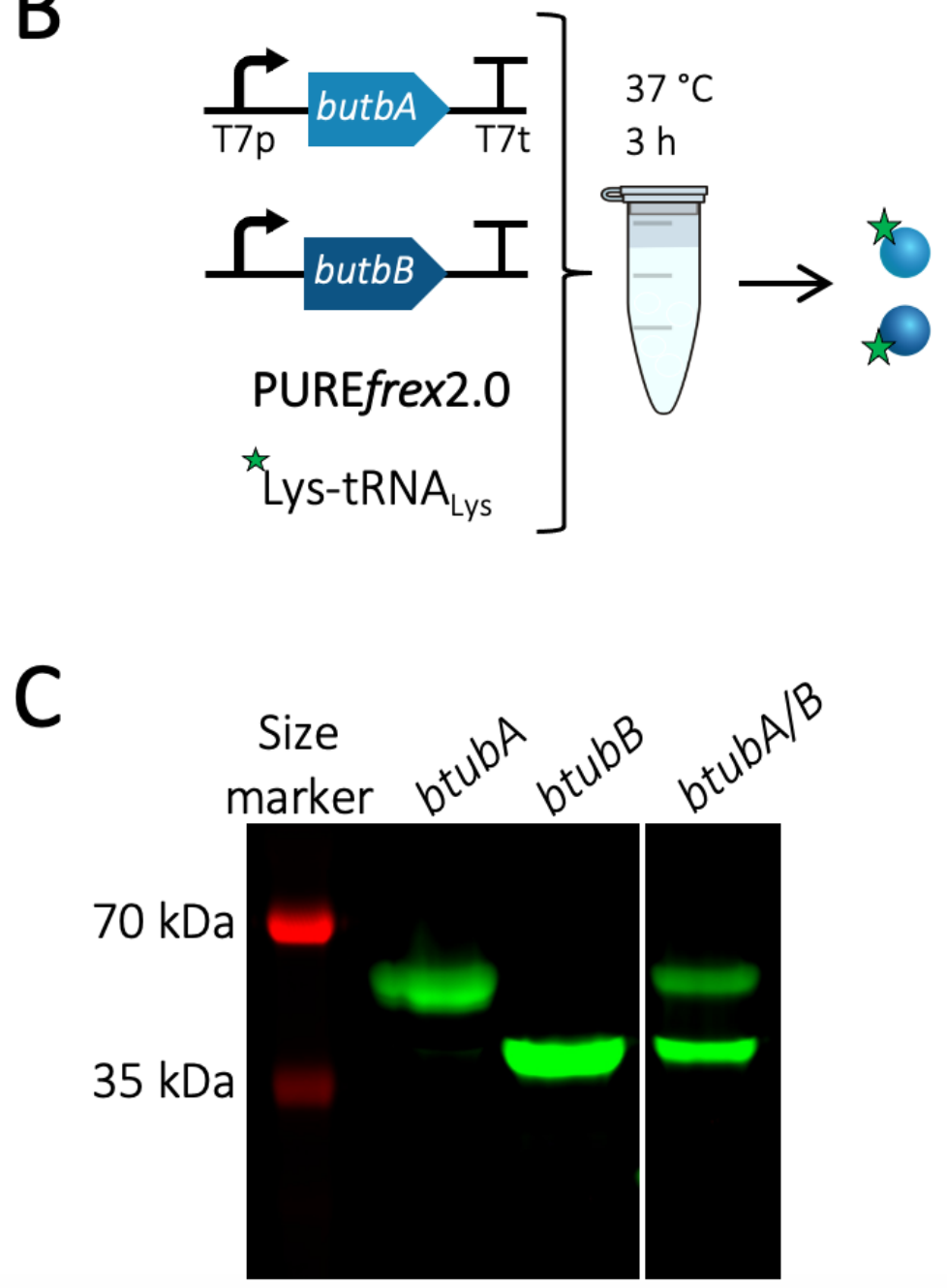

Figure 1: Cell-free expression of BtubA/B. (A) Schematic of a bacterial microtubule consisting of the BtubA/B subunits arranged in four to five protofilaments. The protein BtubC binds to the outside of the filament, predominantly interacting with BtubB. (B) Schematic of CFPS reaction. PUREfrex2.0 was supplemented with the GreenLys reagent to fluorescently label the synthesized proteins through BODIPY-conjugated lysine residues. (C) SDS-PAGE analysis of the gene expression products. The gene names of the expressed DNA templates are indicated on the lanes. The uncropped gel is shown in Supplementary Fig. 2. 
A
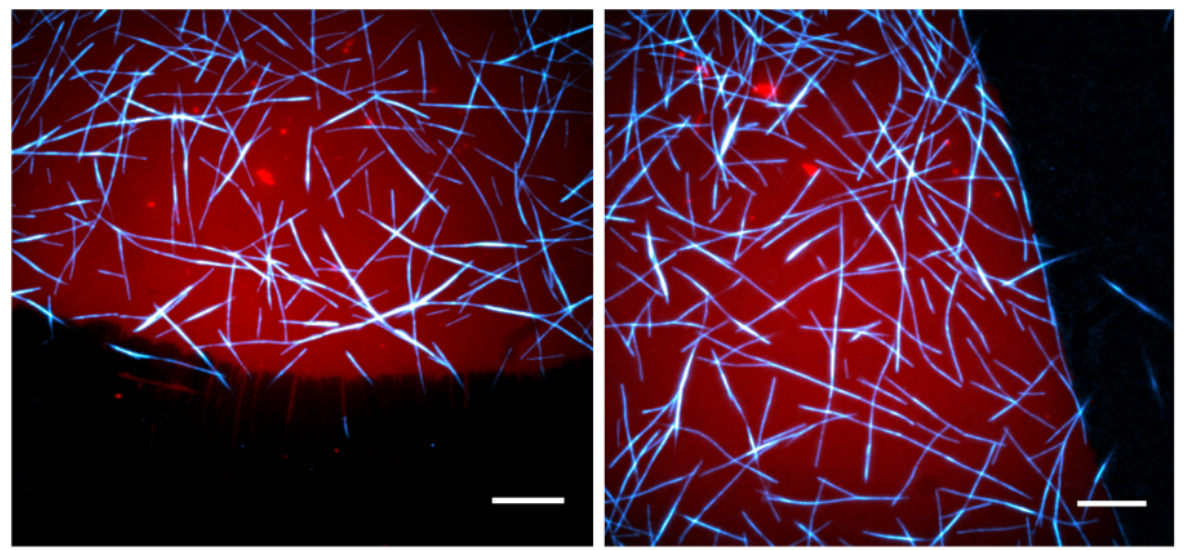

B

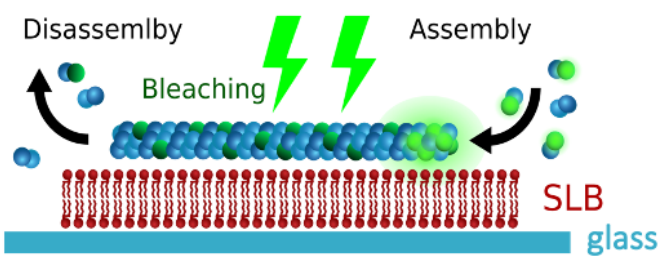

D
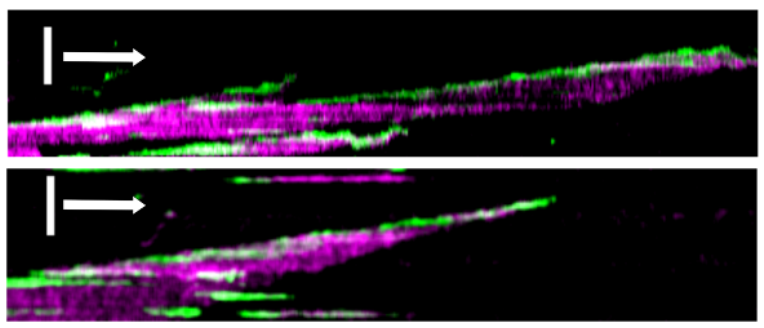

C

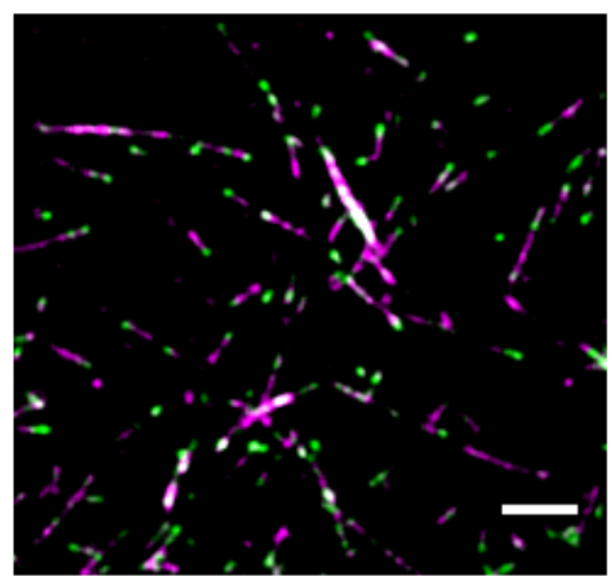

$\mathrm{E}$

Time illumination

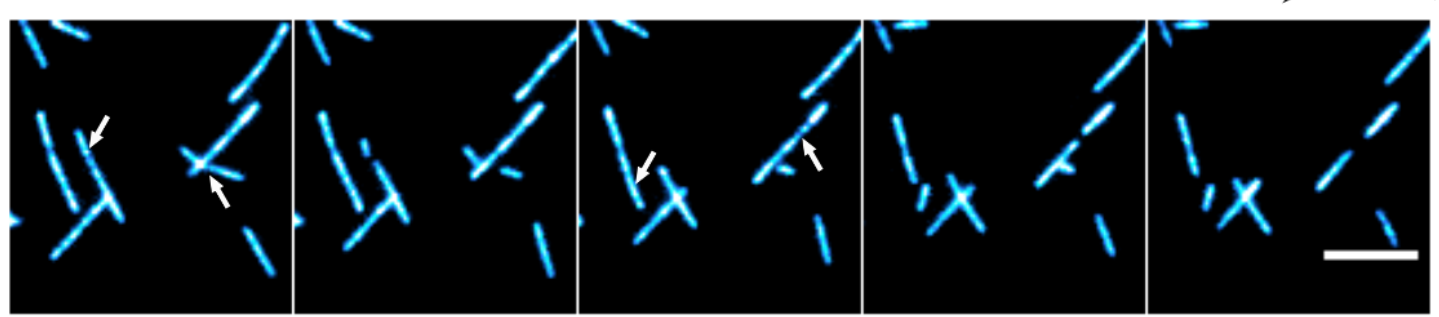

Figure 2: Dynamics of bMTs formed by purified proteins on SLBs. (A) Fluorescence microscopy images showing selective binding of bMTs (cyan, Atto488-BtubA/B) onto an SLB (red, DHPE-TexasRed). Concentration of bacterial tubulins was $2.5 \mu \mathrm{M}$. Scale bars: $10 \mu \mathrm{m}$. (B) Schematic of the dual colour labelling assay to study bMT dynamics on an SLB. Purified BtubA/B proteins labelled with either Atto488 or Atto565 were used. Atto488-labelled bacterial tubulins were bleached continuously during imaging. The only active Atto488 fluorophores are located at the plus end of the filament, where new subunits incorporate. (C) Bleached filaments display fluorescent ends originating from continuous addition of fresh, unbleached Atto488-BtubA/B (green). Atto565-BtubA/B is coloured in magenta. Scale bar: $5 \mu \mathrm{m}$. (D) Kymographs showing bMT dynamics during photobleaching. Vertical scale bar: $5 \mu \mathrm{m}$. Horizontal arrow: 1 min. (E) Time series images showing bMT disassembly events during high-intensity illumination. Arrows indicate the locations at which the filaments break apart. Duration was $120 \mathrm{~s}$ between the first and last image. Scale bar: $5 \mu \mathrm{m}$. 
A
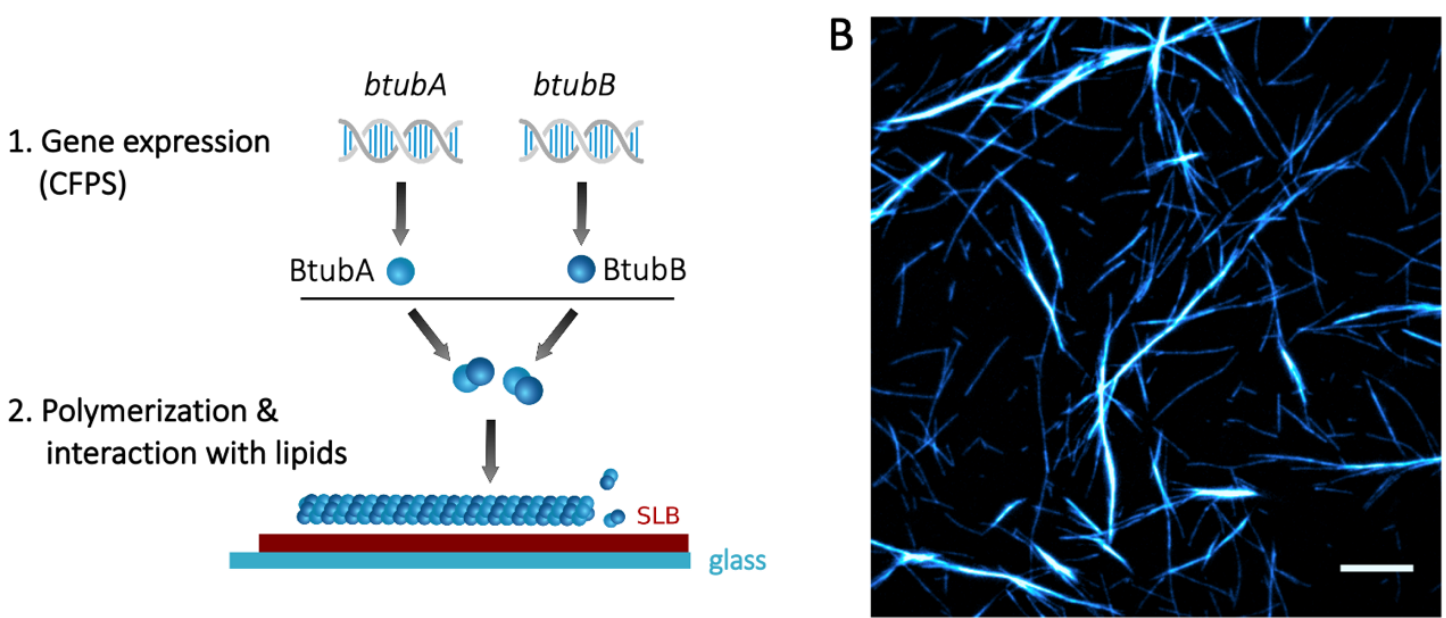

C

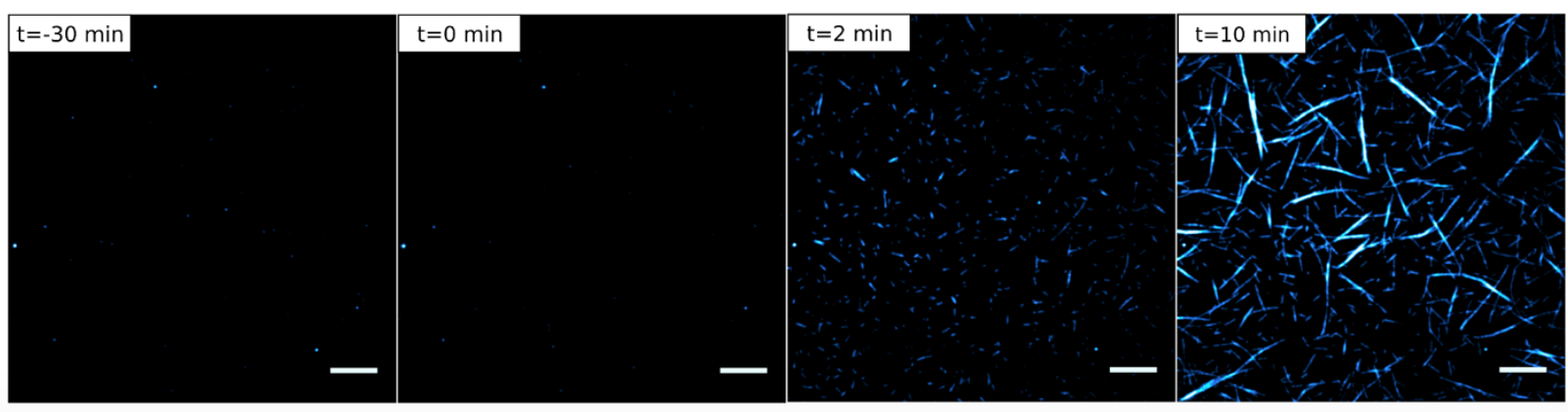

Figure 3: Cell-free expressed BtubA/B assemble into dynamic bMTs on SLBs. (A) Schematic of CFPS and BtubA/B polymerization on supported lipid membranes. (B) Fluorescence microscopy image of cell-free expressed bMTs containing a small fraction of purified Atto488-BtubA/B. Scale bar: $10 \mu \mathrm{m}$. (C) Cell-free synthesized BtubA was mixed with $100 \mathrm{nM}$ Atto488-BtubA/B and incubated on an SLB for $30 \mathrm{~min}$. No filament was observed. At time $t=0$, separately expressed BtubB was added on top of the SLB, triggering immediate formation of short filaments that developed into longer and thicker bundles. Scale bars: $10 \mu \mathrm{m}$. 

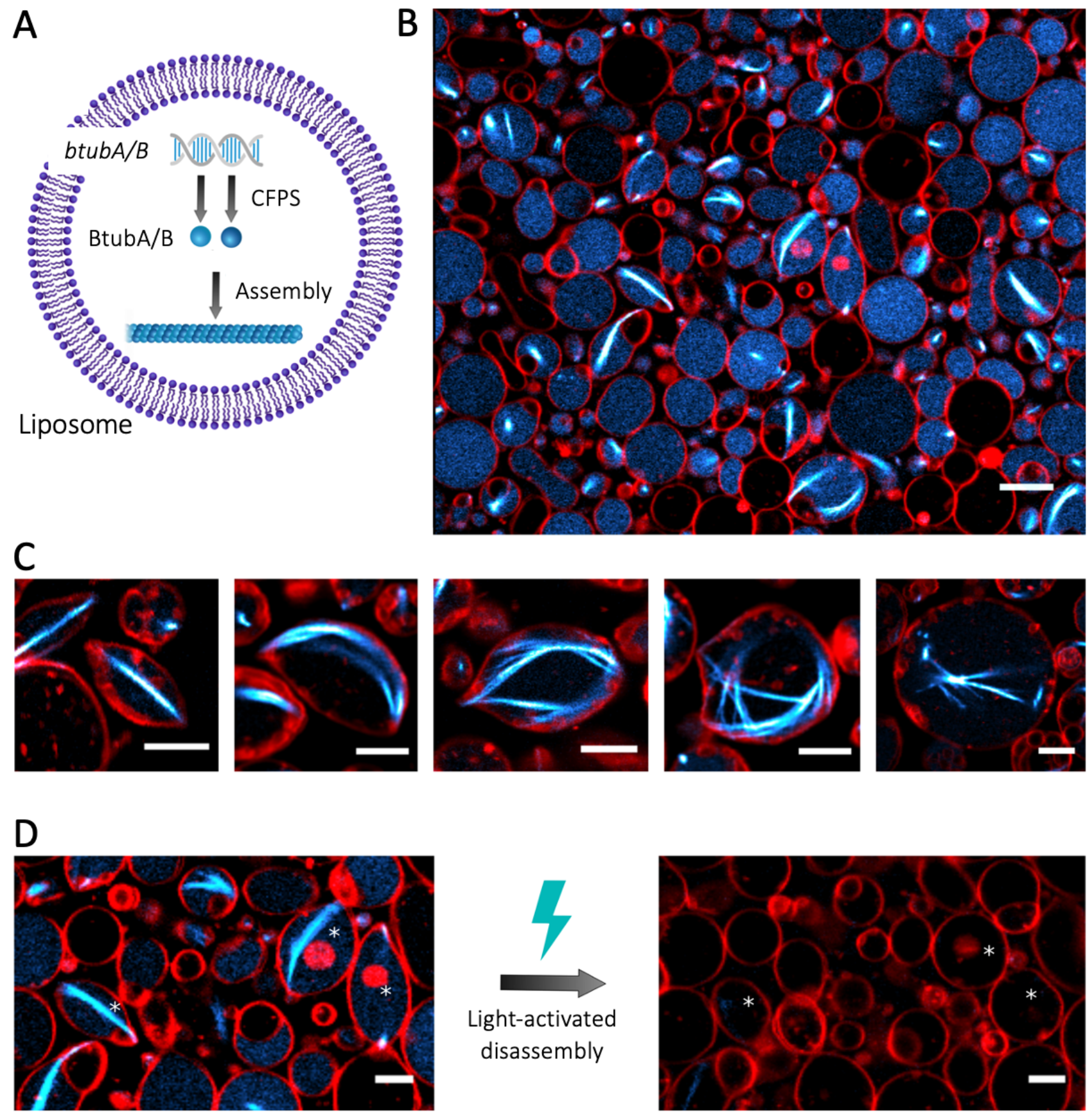

Figure 4: CFPS and assembly of bMTs inside liposomes. (A) Schematic of liposome-compartmentalized gene expression and synthesis of BtubA/B that self-organize into bMTs. (B) Fluorescence confocal microscopy images of liposomes (red, DHPE-TexasRed) with encapsulated Atto488-BtubA/B (100 nM, cyan) after $4 \mathrm{~h}$ CFPS reaction. In situ synthesized bMTs (cyan) are visible in several liposomes. Scale bar: $10 \mu \mathrm{M}$. (C) Examples of different bMT cytoskeletal structures showing clear membrane deformation. Samples were observed after $5 \mathrm{~h}$ of incubation at $37^{\circ} \mathrm{C}$. Scale bars: $5 \mu \mathrm{m}$. (D) Breaking of bMTs and relaxation of liposomes into a spherical shape was triggered by exposing samples to high laser intensity. Asterisks indicate liposomes whose shape was modified by light-activated disassembly of bMTs. Scale bars: $5 \mu \mathrm{m}$. 
bioRxiv preprint doi: https://doi.org/10.1101/2021.06.13.448053; this version posted June 13, 2021. The copyright holder for this preprint (which was not certified by peer review) is the author/funder, who has granted bioRxiv a license to display the preprint in perpetuity. It is made available under aCC-BY-NC-ND 4.0 International license.
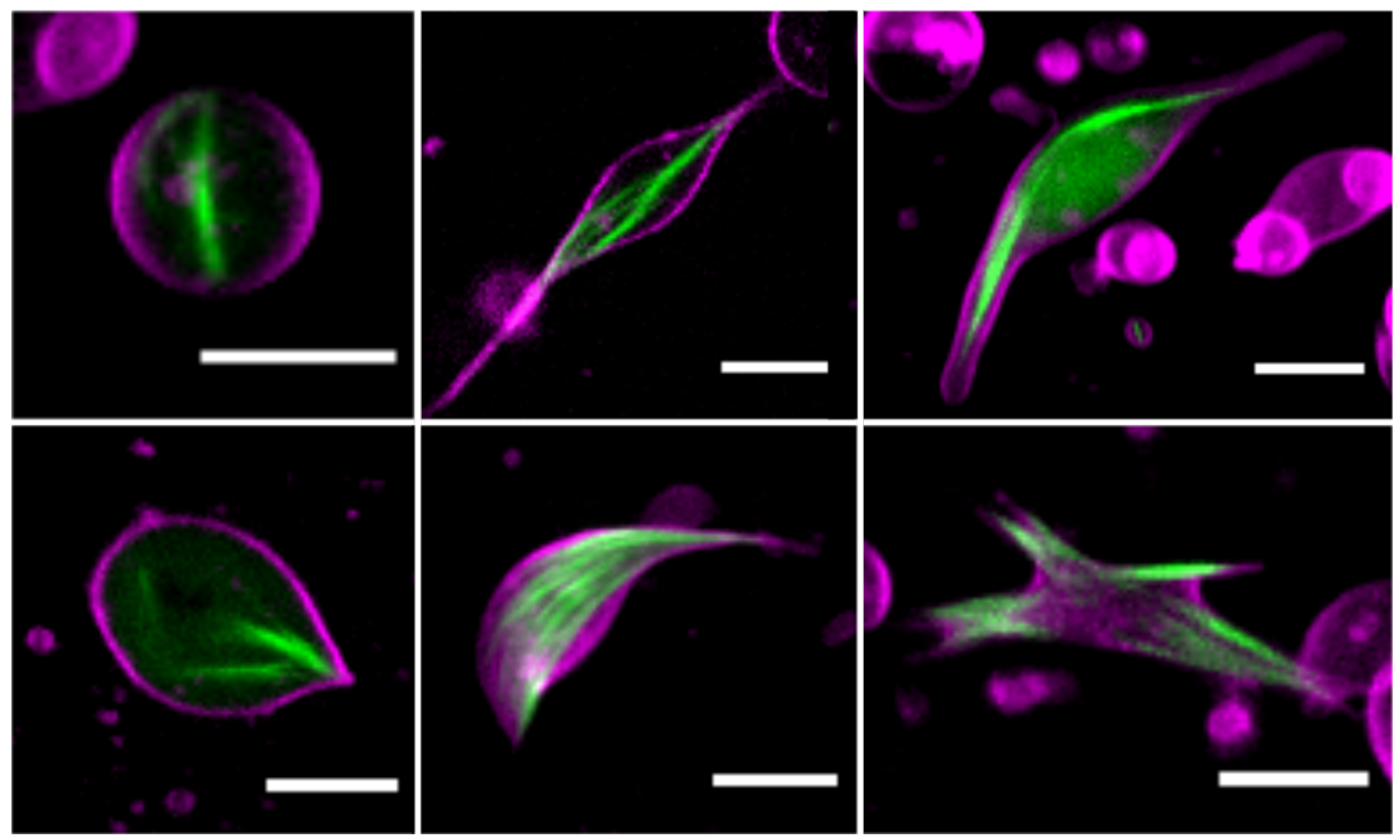

Figure 5: Bacterial microtubules (green, $100 \mathrm{nM}$ Atto488-BtubA/B) formed by $6.6 \mu \mathrm{M}$ of purified tubulins inside liposomes (magenta). Scale bars: $5 \mu \mathrm{m}$. 